\title{
Convergencia y crecimiento en Centroamérica
}

\section{Introducción}

En los últimos años, especialmente desde finales de los ochenta, ha resurgido en la literatura del crecimiento económico el interés en lo que se denomina convergencia entre países o regiones. Si bien existen diferentes definiciones de este fenómeno, especialmente de acuerdo a la metodología utilizada para extraer evidencia empírica acerca de la existencia o no del mismo, puede decirse que existe convergencia entre un grupo de economías (países, regiones, etc.) si las desigualdades en sus niveles de ingreso per cápita tienden a reducirse en el tiempo y el crecimiento de las economías menos desarrolladas tiende a ser mayor y más sostenido en el tiempo que las desarrolladas. Lo anterior implica que, a nivel empírico, se evidencia una relación inversa entre el nivel inicial del ingreso y su crecimiento.

La presunción de la existencia del fenómeno de la convergencia tiene origen teórico en la teoría neoclásica. La contribución fue realizada por Solow en los años 60. El supuesto central del proceso propuesto por el modelo neoclásico es que la relación entre los stocks de factores productivos y el producto nacional puede aproximarse mediante una función de producción agregada que presenta rendimientos constantes a escala en capital físico y trabajo y, por tanto, rendimientos decrecientes en el capital. La existencia de rendimientos decrecientes a escala en el capital (o más general, en varios tipos de capital considerados en el modelo) significa que el producto crece menos que proporcional con el stock de capital. Esto provoca que la productividad marginal de ese factor disminuirá con su acumulación, reduciendo el incentivo a ahorrar y su contribución a un volumen dado de inversión, y la creación de una tendencia a crecer 
cada vez menos a lo largo del tiempo. Este mecanismo genera una predicción de convergencia en rentas per cápita: los países más pobres tendrán un mayor incentivo a ahorrar y una tasa de crecimiento más elevada con la misma tasa de inversión, por lo que tenderán a crecer más rápidamente que los ricos.

Partiendo de la hipótesis según la cual los capitales y la tecnología circulan libremente entre los diferentes países, el modelo de crecimiento neoclásico sostiene que los niveles de ingreso en el mundo convergen en el tiempo. El razonamiento es el siguiente. Dado que los países menos ricos presentan niveles de salarios reales relativamente bajos, sus tasas de rendimiento del capital son relativamente elevadas, lo que implica desplazamientos de los capitales que van de los países más ricos hacia los menos ricos. A medida que se efectúa este proceso de ajuste, las salarios reales en los países menos ricos deberían aumentar para acercarse al nivel observado de los países más ricos.

Esta hipótesis de convergencia es refutada por los nuevos modelos de crecimiento aparecidos a mediados de los años ochenta (Romer (1986), Lucas (1988), Rebelo (1991)). Este conjunto de modelos que niegan la existencia de un estado estacionario, sostienen en cambio que el mecanismo ligado a la dinámica del capital no conoce límite y por lo tanto niegan la existencia de una relación inversa entre la renta inicial y su incremento en el tiempo. Tales modelos, conocidos como de crecimiento endógeno, se fundan sobre un conjunto de supuestos diferentes, pero son caracterizadas por un elemento común: el abandono de la hipótesis de la productividad marginal decreciente, lo cual está asociado a la presencia de externalidades. De tal forma, son los países ricos los que crecen más de prisa y esto hace que el grado de desigualdad aumente sin cesar.

En tal sentido, el estudio de la convergencia viene a cumplir una importante función: Brindar un elemento de contraste esencial en el debate entre la teoría neoclásica y la teoría del crecimiento endógeno. Evidencia a favor o en contra de mecanismos de convergencia entre diferentes economías constituye evidencia a favor o en contra de diferentes teorías del crecimiento económico. Como vimos anteriormente, el debate entre defensores de los modelos de crecimiento endógeno y defensores del modelo neoclásico de crecimiento se ha centrado en gran medida en los supuestos acerca de los rendimientos marginales de los factores y los rendimientos a escala (decrecientes a aquellos y constantes estos últimos en el modelo neoclásico). Dado que el fenómeno de la convergencia está estrechamente relacionado con estos supuestos (ver, por ejemplo, Sala-i Martin, 1996'), el estudio empírico de aquel ha sido utilizado para extraer evidencia de los modelos. 
Otro factor que demuestra la relevancia del estudio de la convergencia es su vinculación con los niveles de bienestar de las diversas economías. La presencia de convergencia indicaría una tendencia, al menos en forma potencial, a una homogenización de los niveles de riqueza de las economías, por lo cual el desarrollo de las economías menos avanzadas podrá ser promovido eliminando el "gap" estructural que lo vincula con el desarrollo. En ausencia de este fenómeno, las diferencias en las riquezas tenderán, al contrario, a quedar inmóviles o incluso a aumentar y su reducción podrá ser obtenida con una intervención pública radical.

En tal sentido, los resultados del estudio de la convergencia se vuelven un punto de discusión relacionado a la política económica a implementar. Por ejemplo, si las diferencias en los niveles de ingreso entre un grupo de economías tiende a disminuir se estará menos preocupado por la creación de programas de asistencia nacionales o regionales, que si éstas tienden a incrementarse. Si se sabe que las regiones que son ahora pobres son las mismas que lo fueron años atrás, a partir de la política económica se les puede permitir escapar de la trampa de la pobreza.

Por otra parte, el alto grado de internacionalización de las distintas economías pone de manifiesto que la situación económica de un determinado país está cada vez más relacionada con la del resto del mundo, lo que justifica la gran atención que se está dando al estudio de los procesos de convergencia. En el caso de las economías centroamericanas, el análisis del proceso de convergencia adquiere mayor relevancia si se tiene en cuenta el proceso de integración económica en que éstas se encuentran. Bajo los supuestos del modelo neoclásico de crecimiento, productividad marginal decreciente del capital y la consideración de la tecnología como un bien público puro (Solow (1956)), los resultados esperados tras la integración son los siguientes: la movilidad de capitales acentúa el proceso de convergencia, ya que aumenta los flujos de este factor de los países más ricos a los pobres. Sin embargo, los nuevos modelos de crecimiento contemplan la existencia de otros posibles mecanismos tales como el proceso de difusión tecnológica (Coe y Helpman (1995)) e incremento de la competencia (Rivera y Romer (1991)); los cuales pueden retardar o impulsar el proceso de convergencia tras la integración.

El presente capítulo sè encuentra organizado de la siguiente manera: En la primera sección se estudian los distintos conceptos y contrastes de convergencia a partir de la metodología empírica que implementan, analizándose los enfoques de convergencia absoluta, condicional y sigma. Así como las nociones recientes de convergencia estocástica y de formación de clubes de convergencia. En la sección II, se hace un recuento de la evidencia empírica encontrada en los principales estudios tanto a nivel 
internacional como regional. La sección III consiste en el análisis preliminar de los datos para el caso de Centroamérica y los resultados empíricos del fenómeno de la convergencia para dicha región durante el período 1950-1995, a partir de distintas formas de comprobación empírica del mismo. Finalmente, la última sección contiene las conclusiones y algunos comentarios finales.

\section{Conceptos y contraste de convergencia}

Los numerosos y variados estudios empíricos sobre la convergencia en la reciente literatura del crecimiento pueden ser clasificados en tres diferentes grupos, dependiendo del enfoque empírico que ellos usan para probar la convergencia. No todos los enfoques econométricos pueden probar todos los aspectos que envuelve el estudio de la convergencia.

El primero es llamado "cross-section analysis". Este examina la regresión de la tasa de crecimiento (promedio) sobre el nivel inicial del ingreso en las diferentes economías. Un coeficiente negativo de la condición inicial es entendido como que las economías pobres crecen más rápido que las ricas y por lo tanto tienden a converger (esto es b convergencia). Esto implicaría que cada economía eventualmente llegaría a ser tan rica como las otras y la dispersión del ingreso entre los países tendería a disminuir a través del tiempo.

Un segundo enfoque de convergencia es el análisis de series de tiempo. Éste prueba la hipótesis de convergencia, en el contexto del análisis de raíces unitarias. En otros términos, el análisis de series de tiempo prueba si las disparidades entre las economías son estacionarias y por lo tanto no divergen en el tiempo.

Un tercer enfoque ha sido recientemente desarrollado por Quah. En este modelo, se estudia la dinámica de la distribución del ingreso a través de las economías. La evolución de la distribución del ingreso entre países envuelve dos clases de dinámicas que ocurren simultáneamente: cambios en el la forma exterior de la distribución y movilidad intra distribución. Este enfoque toma en cuenta ambos, la dimensión tanto del análisis de cross-section y la información de series temporales.

\subsection{Análisis tradicional de la convergencia}

\subsection{1. $\beta$ - convergencia}

De acuerdo con el contraste de b-convergencia (Baumol (1986); Barro y Sala-i-Martin (1991, 1992a); De la Fuente (1994a, 1994b, 1996c, 2000)), se produce este tipo de convergencia cuando la tasa de crecimiento del producto per cápita está inversamente relacionada con el nivel 
de producto inicial. En otras palabras, las economías pobres tienden a crecer más rápido que las ricas. Dentro de este enfoque se hace una distinción entre convergencia absoluta y convergencia condicional.

\subsection{2. $\beta$ - absoluta}

Existe b- convergencia absoluta si los ingresos per cápita de las economías que poseen idénticas características estructurales convergen entre sí en el largo plazo independientemente de sus respectivas condiciones iniciales. Por lo que se debería comprobar en el nivel empírico una relación inversa entre el nivel de ingreso per cápita y su crecimiento de forma tal de generar una tendencia a la igualación de los niveles de producto per cápita de un conjunto de países.

La estimación del coeficiente de b- convergencia absoluta se calcula a partir de una regresión lineal basada en datos de corte transversal de la siguiente manera:

$$
(1 / T) \log \left[\left(Y_{i, 10+T}\right) / Y_{i, 10}\right]=A+B^{*} \log \left(Y_{i, 10}\right)+m_{i}
$$

donde $t_{0}$ y $t_{0}+T$ representan los años iniciales y finales del período de estudio respectivamente e $\mathrm{i}$ es una unidad económica. Y es el ingreso per cápita y $\mathrm{m}$ es el término de error. Por consiguiente, el lado izquierdo es una aproximación del crecimiento promedio de la unidad económica i durante el período $t_{0} y t_{n}+T$. B es un término constante para todas las unidades. Se dirá que existe convergencia si el coeficiente B de esta ecuación es afectado por un signo negativo; lo que implica una relación negativa entre la tasa de crecimiento promedio durante el período de estudio y el logaritmo del ingreso per cápita inicial.

Los estudios recientes de Barro y Sala- i- Martin (1990, 1991 y 1992a) que se centran principalmente en la convergencia entre los estados norteamericanos, utilizan una versión no lineal de esta ecuación. La utilización de los mínimos cuadrados no lineales les permite estimar la velocidad de convergencia de forma directa. La ecuación que estiman se escribe de la siguiente manera:

$$
\log \left(Y_{i, 1+r} / Y_{i, 1}\right) / T=a-\left(1-\exp \left(-b^{\cdot} t\right)\right) / T^{\cdot} \log \left(Y_{i, 1}\right)+m_{i t}
$$

donde la brecha entre las diversas unidades económicas disminuye de forma exponencial a la velocidad de b. Mientras más alto es el valor de b, más rápido se acerca el ingreso per cápita del país más pobre al nivel observado del país rico. 


\subsection{3. $\beta$ - condicional}

La presencia de $b$ convergencia condicional se evidencia si existe una relación negativa entre la tasa de crecimiento promedio del ingreso y su nivel inicial después de tener en cuenta la diversidad en los estados estacionarios de las diversas economías examinadas, es decir, después de tener en cuenta las peculiaridades de las características estructurales. La existencia de convergencia condicional implica que las economías más alejadas de sus respectivos estados estacionarios han crecido, en promedio, más que aquellas economías ubicadas a menor distancia de sus estados estacionarios.

Para la comprobación empírica de este fenómeno, Barro y Sala-iMartin identifican dos maneras de condicionar los datos:

La primera consiste en estimar una versión de la ecuación (1) utilizando datos regionales dentro de un país dado. La omisión de los "fundamentos" en este caso se justifica mediante el argumento de que gran parte de las variables relevantes (incluyendo la estructura institucional, el clima político y social, la política macroeconómica, el nivel de desarrollo tecnológico e incluso las preferencias de los individuos) son comunes a las regiones de un mismo país. Se debería encontrar convergencia absoluta entre este grupo de economías, dado que todas ellas tenderán a acercarse al mismo estado estacionario, y, por tanto, tenderían a acercarse entre ellas.

La segunda consiste en estimar la relación entre crecimiento y renta inicial tras controlar por una serie de variables que se espera capturen los determinantes "fundamentales" del crecimiento en distintos países.

Se dice que un conjunto de economías presenta $\beta$ convergencia condicional si se efectúa una regresión con datos de sección cruzada del crecimiento sobre la renta inicial, manteniendo constante un cierto número de variables adicionales (que actúan de proxy del estado estacionario) y se encuentra que el coeficiente de la renta inicial es negativo.

Para investigar la posibilidad de existencia de convergencia condicional, es necesario estimar:

$$
y_{i, t}=a+B \log \left(Y_{i, t-1}\right)+\phi X_{i t-1}+\mu_{i, t}
$$

Donde $y_{i t}$ es la tasa de crecimiento de la economía entre el período de $\mathrm{t}-1$ y $t, m_{\mathrm{it}}$ es el shock estocástico que recoge las perturbaciones transitorias de la función de producción y $\mathrm{x}_{\mathrm{it-1}-1}$ es un vector de variables que determinan la posición del estado estacionario de cada economía. Se dice que hay convergencia condicional si el signo del coeficiente de $B$ es negativo, una vez se han incluido las variables $X$ en la regresión. 
Los dos tipos de convergencia $\beta$ tienen implicaciones muy distintas. La convergencia b absoluta dentro de un grupo de economías implica una tendencia a la igualación de las rentas per cápita. A largo plazo, el nivel esperado de renta per cápita es el mismo para todos los miembros del grupo, independientemente de su valor inicial. Esto no quiere decir, por supuesto, que la desigualdad llegue a desaparecer por completo, puesto que siempre habrá perturbaciones con efectos desiguales en distintos territorios. Sin embargo, tales perturbaciones tendrán tan sólo efectos transitorios, lo que implica que a largo plazo, debería observarse una distribución fluida en la que los países o regiones cambian su posición relativa con bastante frecuencia.

La convergencia $\beta$ condicional, con diferentes estados estacionarios, daría lugar a una situación muy diferente. Aunque cada territorio tenderá a converger a su propio estado estacionario, éstos podrían ser muy distintos entre sí. Por lo tanto, podrían persistir disparidades importantes entre territorios, incluso a largo plazo, y se observaría una gran persistencia en las posiciones relativas de los mismos. En otras palabras, los pobres tenderían a seguir siéndolo, y los ricos también.

El concepto de $\beta$ - convergencia ha recibido muchas críticas. Algunos autores han sugerido que el test de $b$ (tanto del concepto de convergencia condicional como absoluta) tiene limitaciones. Se dice que el análisis de corte transversal, y particularmente el concepto de b convergencia, está basado en un modelo para una economía "representativa" pero se extraen conclusiones para un grupo de economías. En otras palabras, el proceso de ajuste del modelo de Solow dice que cada economía, después de haberse alejado de su estado estacionario, retorna a él lentamente. Esta es una implicación para un país y por consecuencia no tiene nada que decir acerca de una economía con respecto a otra. Por lo que b convergencia asume implícitamente que el componente permanente del ingreso de cada economía esta bien descrito por una ecuación lineal de tendencia determinística. De hecho, el promedio de la tasa de crecimiento es simplemente la pendiente de la tendencia determinística.

Por ejemplo, Friedman (1992) sostiene que la regresión de corte transversal conlleva al problema clásico del retorno al promedio. También, demostró que, al aplicar esta metodología, contrariamente a lo que podría pensarse intuitivamente, los resultados varían según se utilice el ingreso inicial o el ingreso de fin de período. En el mismo orden de ideas, Quah (1993) compara los resultados de estos tests a los de Galton (1883) relacionados a la estatura de los padres y de sus hijos. Este último había observado que los padres de estatura superior a la media tenían hijos de estatura superior a la media. Sin embargo, la distancia entre la estatura de 
los hijos y la estatura promedio era menos grande que la distancia entre la estatura de los padres y la estatura promedio. El inverso es también verdadero para los padres de baja estatura. Por otra parte, Galton había observado igualmente que, a pesar de esta tendencia a regresar hacia la media, la distribución estadística de la estatura para el conjunto de la población no cambia de una generación a otra, de tal manera que parece improbable que un día todos los hombres tendrán la misma estatura. Quah concluye que la obtención de una relación negativa entre el crecimiento promedio del ingreso per cápita y su nivel inicial no constituye una prueba de existencia de la convergencia.

Entonces, Quah (1993) señala que un parámetro b significativo estimado a partir de una ecuación de corte transversal es compatible con distribuciones de ingresos per capita que tienden a permanecer constantes o incluso a aumentar. De esta manera Quah (1996a) sugiere distinguir claramente entre dos mecanismos: el mecanismo del crecimiento por medio del cual los países desplazan las limitaciones a su crecimiento e incrementan así sus ingresos per cápita y el mecanismo de la convergencia por medio del cual los países tienden a igualar entre sí sus ingresos per cápita. Es el primer mecanismo, argumenta Quah, el que puede estudiarse utilizando el enfoque tradicional, no el segundo.

\subsection{Sigma convergencia}

El concepto de sigma convergencia contribuye a la medición de la convergencia con alguna clase de información acerca de la dinámica de la distribución del ingreso entre los países. De acuerdo con el contraste de $\square$-convergencia (Barro y Sala-i-Martin (1991, 1992a)), ésta se produce cuando la dispersión entre los niveles de renta de distintos países disminuye con el tiempo. La dispersión puede ser medida de diversas maneras. Se escoge la desviación estándar del logaritmo del producto per cápita, tal como lo hacen Barro y Sala-Martin.

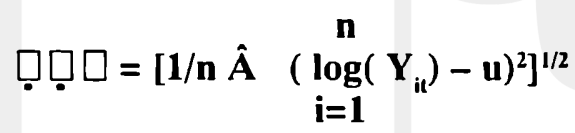

donde $u$ es la media aritmética del $\log \left(Y_{i 1}\right)$.

$\square$ y $\square$ convergencia están relacionadas entre sí pero distan mucho de ser equivalentes. La existencia de algún tipo de convergencia $\square$ es una condición necesaria pero no suficiente para la convergencia $\square$. Mientras que un valdr positivo de $\square$ implicaría una tendencia de $\square$ a crecer sin límite,

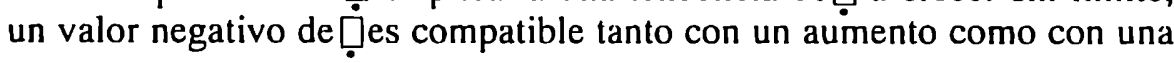


disminución de la dispersión muestral de $y_{\text {il }}$, dependiendo de si el valor inicial de $\varphi_{1}$ es mayor o menor que su valor en el estacionario.

También, la medida de $\sigma$ - convergencia ha sido objeto de críticas. Quah (1993) sostiene que la desviación estándar puede esconder más de lo que muestra; no permite, por ejemplo, detectar la formación de clubes de convergencia, es decir, grupos de economías que tienden a converger entre sí pero no con las economías que integran otros grupos. Además, se dice que la desviación típica es un simple estadístico que no recoge algunos aspectos interesantes de la dinámica de la distribución, es decir, que $\sigma$ - convergencia no toma en cuenta la movilidad intra distribución del ingreso.

\subsection{Análisis de series de tiempo}

$\mathrm{Al}$ análisis de regresiones de corte transversal, le siguieron análisis econométricos de series de tiempo (ver Bemard y Durlauf (1996) y Durlauf y Quah (1998)). Se da la convergencia en este nuevo enfoque cuando las series temporales de los productos per capita relativos (la diferencia entre el producto per capita de cada país y el correspondiente al promedio) no poseen raíces unitarias.

Resulta interesante analizar si las series temporales de los productos per capita relativos de un grupo de países constituye o no un proceso estacionario. En el primer caso, shocks aleatorios que impacten sobre un país generan un diferencial de ingresos transitorio, por lo que se podría hablar de existencia de convergencia mientras que no ocurre lo mismo en el segundo caso donde el efecto de los shocks puede ser permanente.

Si $Y_{m}$ representa la media del logaritmo del ingreso per cápita de un grupo de economías, la convergencia estocástica de la í-esima economía puede ser comprobada estimando la siguiente expresión y probando la estacionaridad:

$$
\Delta\left(Y_{i 1}-Y_{m t}\right)=a+h\left(Y_{i t-1}-Y_{m t-1}\right)+\sum^{k} z_{j} \Delta\left(Y_{1-j}-Y_{m l-j}\right)+e_{i t}
$$

$\mathrm{j}=1$

Si bien esta definición de convergencia es interesante, se debe tener en cuenta los comentarios al respecto de Bernard y Durlauf (1996) en el sentido de que la aplicación de tests de raíz unitaria a los productos per cápita relativos tiende a generar evidencia inconsistente con la generada por la utilización de regresiones de corte transversal para los mismos datos debiéndose esto a los diferentes supuestos que implican acerca de 
la distancia respecto a la distribución límite del proceso. En efecto, los tests de corte transversal llevan implícito el supuesto de que las economías analizadas se encuentran en un proceso de transición hacia sus estados estacionarios mientras que los tests de series de tiempo suponen que las variables analizadas se encuentran cerca de sus distribuciones límite.

\subsection{Análisis de la dinámica de distribución del producto per cápita}

Quah, frente a las limitaciones de los enfoques mencionados anteriormente, propone como alternativa el análisis del comportamiento dinámico de la distribución completa de los ingresos de las economías. Contrariamente al enfoque tradicional y el análisis de series de tiempo, este nuevo enfoque permite detectar la formación de coaliciones entre diferentes economías que generan clubes de convergencia, cuyos integrantes tienden a asemejarse con el paso del tiempo en sus características estructurales, por lo tanto, a converger entre sí.

Por ejemplo, en la siguiente figura, la formación de coaliciones genera el surgimiento de dos clubes de convergencia (el comportamiento dinámico de las economías que integran cada uno de estos está dado por las funciones $\phi[\mathrm{k}(\mathrm{t})]$ y $\phi$ ' $[\mathrm{k}(\mathrm{t})]$, por lo que la distribución de ingresos tiende a convertirse en una distribución bimodal.

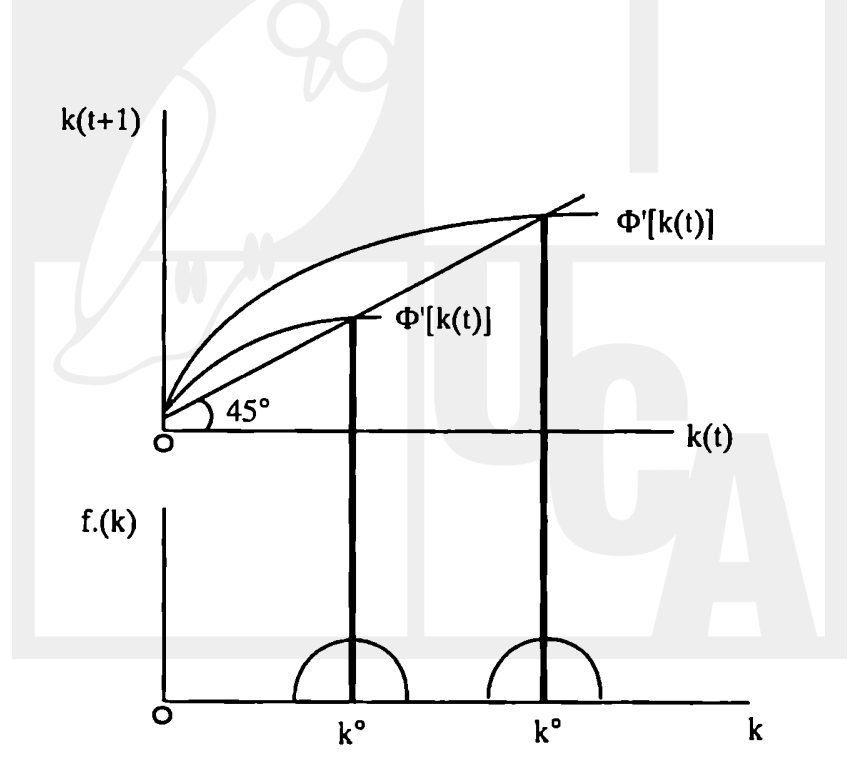

Quah (1993), analizando la evolución dinámica de la distribución de los ingresos de una muestra de países, encuentra evidencia de que estos últimos tienden a concentrarse en dos grupos (ricos y pobres) tendiendo a 
incrementarse, a su vez, la disparidad entre ambos grupos. Cada economía converge a su propio estado estacionario (consistente con la convergencia condicional) pero las interacciones entre las diferentes economías hacen que tiendan a formarse clubes de convergencia en los cuales sus miembros tienden a asemejarse entre sí en sus características estructurales. En este caso, si bien existirá una fuerte relación entre estas características, los diferentes estados estacionarios, la causa última de éstos se encuentra en las variables que determinan la pertenencia a cada club (Quah, 1996b). En otras palabras, las variables que se utilizan para mantener constantes los estados estacionarios en el enfoque "clásico" son, al igual que las tasas de crecimiento, variables endógenas, siendo las variables que explican las diferentes coaliciones entre economías las verdaderas variables explicativas.

\section{Evidencia empírica}

El fenómeno de la convergencia ha sido analizado en muchos estudios. El resultado general que de ellos se deriva, puede resumirse de la siguiente manera: el proceso de convergencia parece haberse limitado al grupo de países más avanzados. En el mundo en su conjunto, se observa un significativo incremento de la desigualdad durante las últimas décadas. A continuación se presentan los resultados de los estudios más relevantes.

Baumol (1986) analiza la convergencia en un grupo de 16 países industrializados durante el último siglo utilizando los datos de Maddison. Dentro de esta muestra, la correlación entre la tasa de crecimiento y el nivel inicial de renta es claramente negativa. Aunque el coeficiente de convergencia es relativamente bajo (un $0,90 \%$ anual), el ajuste de la regresión es muy bueno y la dispersión de la renta per cápita se reduce en dos tercios durante el período 1870-1979. Sin embargo, sus resultados han sido criticados por De Long (1988) quien señala que los países seleccionados por Baumol, son una muestra sesgada; en el sentido que incluye únicamente países que tuvieron éxito al final del período de la muestra, forzando a la convergencia. Cuando De Long incluyó en la muestra otros países que no habían tenido éxito, el coeficiente de convergencia fue menos significativo y más pequeño. Además, la convergencia de los países desarrollados ha sido encontrada por Dowrick y Nguyen (1989), quienes mostraron también convergencia en los niveles de productividad.

En una serie de trabajos, Barro y Sala-i-Martin (1991, 1992 a y b) y Sala-i-Martin $(1995,1996)$ han investigado el patrón de convergencia en muestras nacionales y regionales a partir de una ecuación de convergencia derivada del modelo neoclásico estándar de crecimiento. 
Primero, ellos encontraron evidencia de una correlación parcial negativa entre el ingreso inicial per cápita y su crecimiento ( es decir b convergencia) tanto a nivel nacional como a nivel regional. Sin embargo, existe una diferencia significativa entre los resultados nacionales y regionales: mientras que la convergencia entre una muestra de países emerge sólo cuando se incluyen el nivel de educación, la inversión, la tasa de fertilidad y los índices de inestabilidad política, las regresiones de convergencia a nivel regional dan lugar a un coeficiente negativo sin incluir variables adicionales. Barro y Sala-i-Martin interpretan estos resultados como evidencia que la convergencia entre países es sólo condicional y la convergencia regional, al menos entre los países industriales, es absoluta.

El segundo hallazgo es que la velocidad de convergencia es muy lenta pero estable a través de las muestras. La tasa de convergencia gira alrededor de un $2 \%$ anual. Lo que implica que la duración del proceso de convergencia es de 35 años, por lo que la eliminación de las disparidades del ingreso podría tomar varias décadas.

Barro (1991) no encontró evidencia de b convergencia absoluta para una muestra de 98 países en el período de 1960-1985. Sin embargo, sus resultados para la Unión Económica Europea con datos para 1950-1985 confirmaban la hipótesis de b convergencia absoluta. Para una muestra de alrededor de 100 países observados en los años 1960-1990, este mismo autor volvió a encontrar una fuerte evidencia de la existencia de convergencia condicional (Barro, 1997).

Otra fuente de verificación empírica, puede encontrarse en Sala-i-Martin (1996). Este autor muestra que, en los países de la OCDE, hay evidencia de beta absoluta y sigma convergencia para el período de 1950-1990. Sin embargo, encontró divergencia en una muestra de 110 países, para el mismo período y para cada una de estas medidas.

También, se han realizado estudios para determinar la convergencia entre regiones para determinados países. Sala-i-Martin (1996) reportó evidencia para beta convergencia absoluta en 48 estados norteamericanos para el período 1980-1990. Encontró similares resultados entre las prefecturas japonesas para el período 1930-1990 y entre los grupos de regiones de cada uno de los cinco países europeos (Alemania, Francia, Italia, Reino Unido y España) para el período 1950-1990.

Los resultados de Barro y Sala-i-Martin fueron confirmados por otros estudios que aplican sus técnicas a muestras de regiones. Coulombe y Lee (1993, 1996), Dolado et al. (1994), Shioji (1996) y Svensson (1997) entre otros, han investigado el proceso de convergencia en Canadá, España, Japón y Suecia. Aunque sus estimaciones de la tasa de convergencia 
varía un poco, sus resultados son generalmente consistentes con la hipótesis de la lenta convergencia b absoluta.

Los estudios empíricos que utilizan la metodología tradicional (ecuaciones a la Barro), han generado evidencia consistente con la convergencia entre economías con características estructurales semejantes. Sin embargo, enfoques alternativos como el de Quah han generado nuevas controversias en lo referente a la metodología a usar para extraer evidencia empírica a cerca del fenómeno de la convergencia. En una serie de trabajos, Quah (1993, 1995a, 1995b, 1996a, 1996b, 1997) aplica el análisis de la dinámica de la distribución de los productos per cápita de distintas economías y demuestra la existencia de fenómenos imposibles de ser captados con el enfoque tradicional, por ejemplo, la existencia de clubes de convergencia.

Por su parte, De la Fuente (2000), cuestiona la evidencia de convergencia absoluta, incluso dentro del grupo de los países más avanzados. Para él, existe muy poca evidencia en favor de la hipótesis de que la diferencia de rentas entre los países ricos y los pobres tiende a reducirse de forma espontánea con el paso del tiempo. Además, el pésimo ajuste de las regresiones de convergencia no condicionada, sobre todo en muestras amplias, sugiere que las diferencias iniciales en niveles de renta distan mucho de explicar convincentemente la variación observada de la tasa de crecimiento entre países. Por tanto, resulta necesario extender las ecuaciones de convergencia utilizadas hasta el momento, incorporando a las mismas, variables adicionales que podrían ayudar a explicar la variada experiencia de crecimiento de los distintos países.

Las pruebas de convergencia estocástica han dado lugar a resultados conflictivos. Neven y Gouyette (1995) encontraron que, en los países de la Unión Europea en el período 1975-1990, los ingresos relativos eran estacionarios; lo cual interpretaron como evidencia de que las disparidades económicas no estaban aumentando. Evans y Karras (1993) no encontraron evidencia de convergencia en una muestra de 53 países, excepto cuando la muestra se restringía a los 17 países más ricos, mientras que, en su estudio de 1996, encontraron evidencia de convergencia estocástica en los 48 estados contiguos de los Estados Unidos. Sin embargo, Carlino y Mills (1993) concluyeron que existían raíces unitarias en las series temporales del ingreso de 5 de los 8 estados de ese país.

Entre los estudios de convergencia aplicados a países latinoamericanos, se pueden citar el de Verspagen (1995) y José de Gregorio (1992). Verspagen estudió la convergencia en seis países latinoamericanos (Argentina, Brasil, Chile, Colombia, México y Venezuela) para el período 1903-1987. Encontró que en esos países no existe una tendencia clara y el comportamiento de cada país es irregular. De la misma manera, José 
de Gregorio, no encontró prueba de convergencia (incondicional) del ingreso per cápita en los países de América Latina.

\section{Resultados para la región centroamericana (1950-1995)}

\subsection{Análisis preliminar de los datos}

La verificación de la convergencia puede ser precedida por una presentación de los datos relativos al crecimiento de los países en el período 1950- 1995. Todos los datos del ingreso per cápita fueron tomados del "Penn World Table" de Summer y Heston (versión 5.6) y complementado para el período 1990-1995 con la base del Banco Mundial.

En la tabla 1 se presentan los datos del Producto per cápita para los diversos países con sus respectivas tasas de crecimiento. Como puede observarse, Centroamérica se caracteriza por una gran desigualdad en el nivel de ingreso per cápita de los países que la integran. Efectivamente, en 1950 el producto per cápita del país más rico (Guatemala) era 1.56 veces mayor que el correspondiente al país más pobre en aquel año (Honduras), diferencia que, luego de cuatros décadas, se incrementó a 3.13 veces, como lo indica el cociente entre los productos per cápita de Costa Rica y Nicaragua, el país más rico y el más pobre respectivamente en el año 1995.

Simultáneamente, existe una reducida movilidad del ingreso de los distintos países, es decir, no presentan grandes cambios en su posición relativa en 1995 en comparación al año inicial. El Salvador ha conservado su posición. Honduras y Costa Rica ascendieron una posición, situándose en la cuarta y primera posición respectivamente. Nicaragua y Guatemala se han desplazado una posición, pero en sentido contrario.

Tabla 1

Indicadores de crecimiento económico en Centroamérica

\begin{tabular}{|c|c|c|c|c|c|c|c|c|c|c|}
\hline \multirow[b]{2}{*}{ Puis } & \multicolumn{3}{|c|}{ Produclo Per cipita } & \multicolumn{4}{|c|}{ Crccimicnlo } & \multicolumn{3}{|c|}{ Kanking } \\
\hline & PIBSTा & PIBGT & PIBप5 & $\begin{array}{l}\text { GThat } \\
\text { S(1/95 }\end{array}$ & $\begin{array}{l}\text { Global } \\
\text { 6ily95 }\end{array}$ & $\begin{array}{l}\text { Anutal } \\
50-95\end{array}$ & $\begin{array}{l}\text { Anual } \\
60-95\end{array}$ & T45ा & Ty95 & $\begin{array}{l}\text { Crec. } \\
501 / 45\end{array}$ \\
\hline Costi Rica & 1457 & $2(1) 6$ & 38115 & 161.1 & 81.5 & 2.2 & 1.8 & 2 & 1 & 1 \\
\hline El Salvador & 12116 & 1427 & 2130 & 76.6 & 49.3 & 1.3 & 1.2 & 3 & 3 & 2 \\
\hline Gualcmilia & 1532 & | 6601 & 23.357 & 5.3 .8 & 4211 & 1.0 & 1.0 & 1 & 2 & 3 \\
\hline Honduras & 98ा & TRBप & T385 & पार & 33.3 & $\pi .8$ & 01.9 & 5 & 4 & 4 \\
\hline Nicaragua & 1152 & 1696 & 1215 & 5.5 & -24.4 & 0.4 & -05 & 4 & 5 & 5 \\
\hline C. A & 1035 & 1542 & 2118 & I04.6 & 37.4 & $1.1^{\circ}$ & 0.97 & & & \\
\hline Fucnic: Cálc & propios & & & & & & & & & \\
\hline
\end{tabular}

Detrás de estos fenómenos de desigualdad e inmovilidad, se encuentra el fenómeno del crecimiento económico. Como se desprende de la tabla 1, el producto per cápita regional creció entre los años 1950 y 1995 a una 
tasa anual promedio de $1.1 \%$, lo que permitió un aumento del mismo de un $104.6 \%$ durante el periodo mencionado. De los países analizados, sólo Costa Rica ha incrementado más de un $100 \%$ su producto per cápita. Guatemala, El Salvador y Honduras crecieron a tasas que les permitieron incrementarlo entre un $40 \%$ y un $80 \%$ en el mismo periodo. Nicaragua aumentó solamente en $5.5 \%$ su producto per cápita durante todo el período pero, para el subperíodo 1960-1995, decreció a una tasa anual promedio del $-0.5 \%$ y perdió un $24.4 \%$ de su producto per cápita original.

Resulta evidente, al observar las distintas tasas de crecimiento presentadas en la tabla 1, que junto con las grandes desigualdades mencionadas anteriormente en sus niveles de desarrollo, los países centroamericanos se han caracterizado por crecer a tasas muy dispares. En efecto, la diferencia entre la mayor tasa de crecimiento anual promedio en el período 1950-95 (2.2) correspondiente a Costa Rica y la menor de ellas (0.4\%) correspondiente a Nicaragua es de 1.80 puntos porcentuales, una diferencia considerable si se tiene en cuenta que un país creciendo a ese ritmo duplica su producto per cápita en 39 años.

Debido a la gran heterogeneidad de los niveles de desarrollo de los países analizados, para alcanzar a Costa Rica, el país más rico en 1995, Guatemala, El Salvador y Nicaragua deberían haber crecido a una tasa anual promedio mayor al $2.0 \%$ y Honduras debería haberlo hecho a una tasa mayor que $3.0 \%$. Lo anterior indica que aún en el caso de que los países más pobres de Centroamérica crecieran en las próximas décadas a tasas mayores que los más ricos, se requerirían varias generaciones para observar una convergencia entre los mismos. Más aún, si se tiene en cuenta que en el año 1995, el mayor producto per cápita de Centroamérica (los US \$ 3805 de Costa Rica) representaba tan sólo el $20 \%$ del correspondiente a Estados Unidos (con sus US\$ 18975) y el menor de ellos (los US\$ 1215 de Nicaragua) un insignificante 6.40\% de este último, resulta evidente la importancia que tiene el estudio del crecimiento económico al momento de pensar las estrategias a seguir en estos países.

\subsection{Convergencia absoluta sigma y beta}

Otra forma de verificación de la convergencia que puede realizarse es la relativa a la convergencia absoluta que consiste en el análisis de la relación entre las tasas de crecimiento y producto inicial. Tal forma de convergencia puede ser analizada de dos modos. El primero está constituido por la confrontación entre una medida de dispersión de los valores del Producto Per Cápita en las áreas de estudio, calculados mediante un índice de variabilidad del producto, evaluando si tal índice se reduce en el tiempo. Esta forma de convergencia es denominada por Barro-Sala-i- 
Gráfico 1

Desviación estándar del logaritmo del producto per cápita

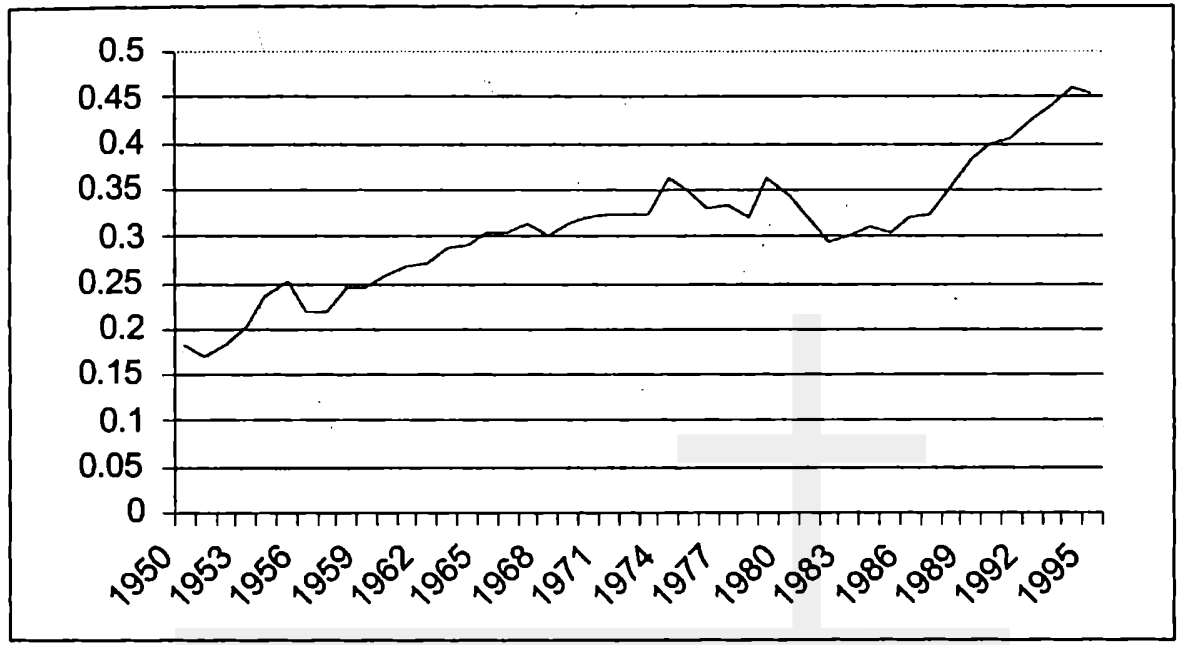

Martin sigma convergencia y proponen medir la variabilidad del producto mediante la desviación estándar.

Se calculó la desviación estándar del logaritmo natural de los niveles de ingreso real per cápita de los países para cada uno de los años. Como puede apreciarse en el gráfico, $s(t)$ presenta una tendencia creciente en el período/de estudio. El nivel de este índice en 1995 representó un incremento de $150.9 \%$ de su nivel en 1950 . Esto puede explicarse por el hecho de que Costa Rica, el país más rico desde 1952, experimentó tasas de crecimiento más dinámicas que los otros países de la región.

Durante los años sesenta, que corresponden al período de funcionamiento del Mercado Común Centroamericano (MCCA), se nota una tendencia creciente en el comportamiento de la dispersión del ingreso entre los países. Contrariamente a la experiencia de los países de la Unión Europea, el incremento del comercio intrarregional no ha dado lugar a una disminución de las disparidades en Centroamérica.

El índice de dispersión siguió creciendo en los años setenta. Sin embargo presentó una leve caída en el primer quinquenio de los ochenta, la cual se explica más por la crisis que experimentaron todos los países que por un mayor dinamismo de los países pobres de la región. La divergencia entre los países se acentuó durante los años noventa a pesar de la recuperación del comercio intrarregional. 
Para dimensionar la magnitud de la disparidad entre los países de la región centroamericana, siguiendo a Cáceres (1998), se compara el valor del índice con los datos presentados por Sala-i-Martín (1994). En 1950 la dispersión de Centroamérica era $33 \%$ menor que la de los estados norteamericanos, pero para 1988, ésta era $84 \%$ mayor. El valor del índice de la región en 1990 era 1.48, 1.81, 3.33 veces el valor de las regiones de Italia, España y Reino Unido respectivamente.

El análisis de sigma convergencia puede ser completado por el de b convergencia absoluta. Para ello, se usará una ecuación a la Barro. Este método utiliza datos de corte transversal y examina la relación que existe entre las dos variables siguientes:

- El nivel de inicio del PIB per cápita de cada país (o sea el de 1950), y

- La tasa de crecimiento promedio del PIBP de cada país sobre el conjunto del período (o sea 1950-1995).

En la tabla 2, los datos del PIB per cápita al inicio del período han sido clasificados en orden creciente, partiendo de Honduras, el nivel más bajo, hasta Guatemala, el más elevado. La correlación entre estos dos vectores de observaciones acusa un valor de 0.55 . Esta correlación positiva no es compatible con la hipótesis de la convergencia.

\section{TABLA 2}

Comparación de la tasa de crecimiento promedio del PIB per cápita entre 1950-1995 y su nivel inicial

\begin{tabular}{|l|c|c|}
\hline País & PIB per cápita de 1950 & $\begin{array}{c}\text { Crecimiento promedio } \\
1950-1995\end{array}$ \\
\hline Honduras & 981 & 0.8 \\
Nicaragua & 1152 & 0.4 \\
El Salvador & 1206 & 1.3 \\
Costa Rica & 1457 & 2.2 \\
Guatemala & 1532 & 1.0 \\
\hline
\end{tabular}

La estimación de la ecuación a la Barro, mediante el método de mínimos cuadrados no lineales, es presentada en el siguiente gráfico. La verificación de la convergencia, según la especificación de la ecuación (2) implica que el parámetro b resulta positivo; cuando esto sucede se debe concluir que entre los datos existe una relación inversa entre el ingreso 
inicial y la tasa de crecimiento. En el caso centroamericano, se observa que la relación entre el crecimiento promedio y el nivel de ingreso es positiva.

Gráfico 2

Relación entre el PIB inicial y el crecimiento (1950-1995)

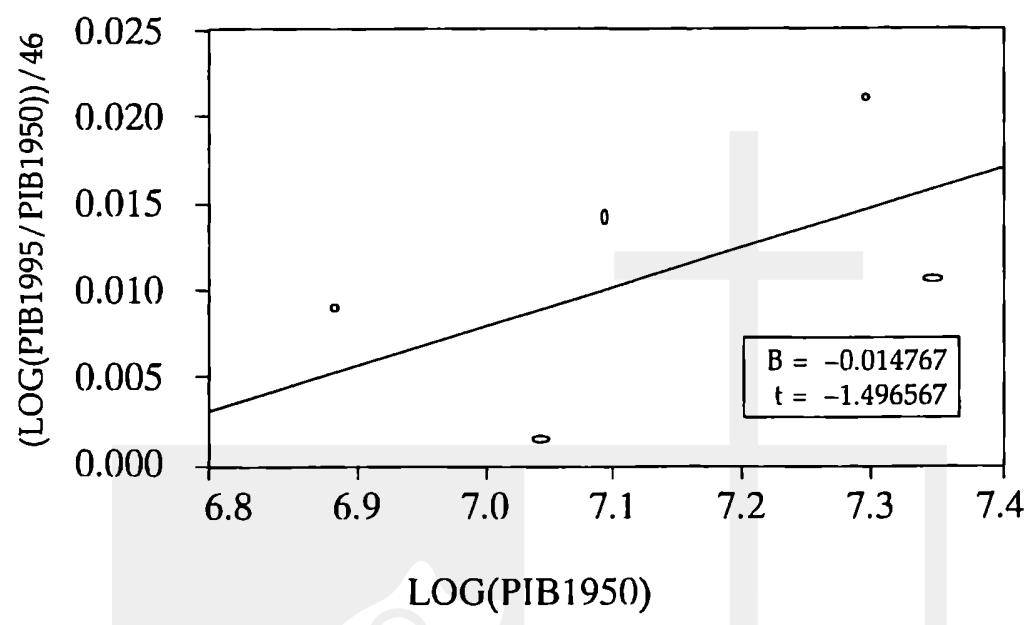

Los resultados de la regresión no son alentadores, ya que el coeficiente $b$ es negativo y no es estadisticamente diferente de cero. Por lo descrito, no podemos hablar de la presencia de b convergencia absoluta a nivel del PIB per cápita en el período mencionado. Lo cual evidencia que los cinco países centroamericanos no constituyen una región homogénea, ya que las peculiaridades de las distintas características estructurales (el marco institucional, el clima político y social, la política macroeconómica y el nivel de desarrollo tecnológico) juegan un papel importante en la determinación de las disparidades entre los niveles de ingresos de los países. Lo que refleja que los países no tienden a converger a un mismo estado estacionario:-

Nótese que este resultado es consistente tanto con modelos de crecimiento endógeno (en los cuales la tasa de crecimiento durante un período determinado no está sistemáticamente correlacionada con el nivel de ingreso al comienzo de dicho período) como con el modelo neoclásico de crecimiento (en el cual ambas variables están correlacionadas negativamente sólo sí los estados estacionarios son semejantes). 


\subsection{Convergencia condicional}

Dado que no existe evidencia en favor de la hipótesis de que la diferencia de rentas entre los países centroamericanos relativamente ricos y los pobres tiende a reducirse de forma espontánea con el paso del tiempo, resulta necesario extender las ecuaciones de convergencia utilizadas hasta el momento teniendo en cuenta los diversos estados estacionarios de las economías examinadas; con el fin de explicar la variada experiencia de crecimiento de los distintos países.

Tal como se indicó anteriormente, la presencia de convergencia condicional ha sido tradicionalmente comprobada a partir de la estimación de la ecuación (3). Sin embargo este método ha sido objeto de críticas (Marcet, 1994; Gorostiaga, 1999; De La Fuente, 2000); ya que puede generar algunos problemas como los siguientes:

- Las variables condicionantes habitualmente utilizadas en la literatura podrían no capturar todas las diferencias importantes entre países, dado que resulta difícil identificar las variables relevantes y obtener los datos necesarios. La exclusión de las características propias de cada país o región en la estimación crea un sesgo de variables omitidas, lo que podría explicar las bajas tasas de convergencia que se han encontrado tradicionalmente.

- Los parámetros estimados pueden estar sesgados cuando los regresores incorporados en la ecuación están correlacionados con los que se han omitido. En otras palabras, se pueden controlar algunas de las diferencias tecnológicas entre países en el término de error sólo si éstas no están correlacionadas con las variables que se han incluido, tales como la inversión y el crecimiento de la población.

Lo anterior indica que el modelo de Barro y Sala-i-Martin podría estar mal especificado y que la estimación del parámetro de convergencia podría estar sesgada. La aparente convergencia lenta hacia un estado estacionario (en el caso de las regiones) podría deberse a una especificación incorrecta y a nivel de países podría existir un problema similar en la medida en que las variables condicionantes utilizadas no capturen todas las diferencias relevantes entre ellos.

La solución estándar para evitar este problema es la utilización de técnicas de panel de datos en función de controlar los efectos fijos no observados tanto a nivel nacional como regional. El procedimiento simple consiste en introducir variables dummies para cada uno de los países, esto es equivalente a estimar una ecuación calculada en el tiempo para cada país o región en la muestra. 
La utilización de datos de panel implica disponer de muestras formadas por las observaciones de $\mathrm{N}$ unidades muestrales (países en nuestro caso) en t instantes. La especificación general de un modelo econométrico de datos de panel es:

$$
g_{i 1}=m+B X+n_{i 1}+w_{i}+u_{i 1}
$$

En la que $\mathbf{g}_{\mathrm{it}}$ es la variable endógena, $\mathbf{i}$ indica la unidad muestral y $\mathbf{t}$ el periodo, $\mathbf{m}$ es un intercepto general, $\mathbf{X}_{\mathbf{t}}$ es un vector de variables explicativas observables, $\mathbf{n}_{\mathbf{i}}$ es un efecto no observable propio de cada unidad muestral e invariable en el tiempo, $w_{1}$ es un efecto propio de cada período y común a todas las unidades muestrales, $y \mathbf{u}_{11}$ es el término de error aleatorio clásico.

Básicamente, existen dos maneras de estimar la ecuación (6). Una considera que $n_{i} y w_{1}$ son fijos, es decir, son interceptos propios de cada unidad muestral y de cada periodo respectivamente. Este modelo es Ilamado de " efectos fijos" (fixed effects), el cual se estima a partir de una regresión de mínimos cuadrados ordinarios de $\mathbf{g}_{\mathfrak{t}}$ sobre $\mathbf{X}_{\mathrm{it}}$ con variable dummy para cada país (LSDV). La otra consiste en asumir que $n_{i}$ y $w_{1}$ son realizaciones de una distribución de probabilidades subyacente, de tal manera que se forma un término de error compuesto: $v_{i t}=n_{i}+w_{1}+u_{i i}$, este modelo es llamado de efectos aleatorios (random effects).

El método más adecuado para estimar regresiones de crecimiento es el de efectos fijos porque el método de efectos aleatorios presenta la desventaja de que el efecto no observado puede estar correlacionado con los regresores (Maria Teresa Guin-Siu (1999)); lo que implica que no corrige los problemas que puede presentar la estimación de la ecuación tradicional que mide la convergencia condicional. A pesar de las bondades del enfoque de panel, no deja de ser objetable. Su gran desventaja es que no permite identificar los factores que explican las diferencias entre los equilibrios a largo plazo de distintos territorios. La significatividad de las dummies sugiere que los países, son muy distintos entre sí pero no se sabe en qué.

Para estimar la convergencia a partir de la técnica de panel de datos, se utiliza la siguiente ecuación:

$$
y_{i t}=a_{i}+b^{*} \log \left(Y_{\text {oit }}\right)+e_{i t}
$$

donde $y_{i 1}$ es la tasa de crecimiento promedio de cada país para subperíodos de tres años calculada a partir de la diferencia logarítmica, ai es una 
constante que incluye los efectos específicos de cada país y $\log \left(\mathrm{Y}_{0 \mathrm{in}}\right)$ es el logaritmo del ingreso inicial para cada subperíodo. Nótese que este modelo podría extenderse para incluir los efectos propios de cada período (time-specific effects), pero no es necesario dado que las observaciones fueron agrupadas en subperíodos de tres años y por lo tanto la variabilidad en el tiempo es capturada por los mismos regresores.

En primer lugar, se estimará una versión incondicional de esta ecuación asumiendo un intercepto común para todos los países, es decir, se probará la existencia de convergencia absoluta. Luego, se estimará esta misma ecuación usando mínimos cuadrados ordinarios con variables dummies para tomar en cuenta los efectos fijos de cada país, probándose de esta manera la existencia de b convergencia condicional. La heterocedasticidad de las regresiones es corregida utilizando White Correction.

TABLA 3

Estimación de la velocidad de convergencia en la región centroamericana utilizando datos de panel*

\begin{tabular}{|c|c|c|}
\hline Método: Pooled Least Squares & Sin fixed effects (1) & Con fixed effects (2) \\
\hline B & -0.008885 & -0.046761 \\
Desv.Estándar & 0.010631 & 0.019299 \\
Estad. T & -0.835782 & -2.422956 \\
Probabilidad & 0.4060 & 0.0180 \\
\hline Observaciones incluidas: 15 & \\
Número Total observaciones en el panel (balanceado): 75 \\
* Período 1950-1995, Sub-periodos de tres años. \\
\hline
\end{tabular}

Los resultados para las dos versiones de la ecuación se presentan en la tabla 3. Como puede observarse, al omitir las variables que detectan los efectos específicos de cada país (dummies) en la regresión (1), los resultados son consistentes con la estimación que se obtuvo de $B$ convergencia absoluta a partir de la metodología de Barro. El coeficiente $\beta$ tiene el signo esperado pero no es estadísticamente diferente de cero.

La inclusión de variables ficticias en la regresión (2) permite detectar efectos específicos y ofrece una visión muy distinta del proceso de convergencia. El coeficiente $B$ negativo y significativo indica la existencia de convergencia $B$ condicional, lo que constituye evidencia a favor del modelo neoclásico de crecimiento y además es consistente con la hipótesis de formación de clubes de convergencia desarrollada por Quah. 
En términos del modelo neoclásico, los países convergerían a diferentes estados estacionarios reduciendo las brechas que los separan de éstos a una tasa de aproximadamente $4.6 \%$ anual. Lo que apunta hacia una conclusión diferente a la de Barro y Sala-i-Marti, es decir, hacia una velocidad de convergencia condicional mayor que $2 \%$. Resultados similares han sido obtenidos por otros autores, al utilizar técnicas de panel e introducir efectos fijos. Marcet (1994) y Canova y Marcet (1995) encontraron tasas de convergencia alrededor del $11 \%$ para una muestra de países de la OCDE y $23 \%$ para las regiones de la Unión Europea. De la Fuente (2000), en el caso de las provincias españolas, al estimar la tasa de convergencia incondicional encontró una tasa del $2.2 \%$; la cual se incrementó a un $8 \%$ al considerar efectos fijos. En la misma línea, Islam (1995) obtiene estimaciones de la tasa de convergencia que oscilan entre $4.3 \%$ y 9.3\% correspondiente a varios países.

Tal como sugiere De la Fuente $(1995,1996,1998,2000)$, una tasa de velocidad de convergencia mayor podría estar indicando que además de la existencia de rendimientos decrecientes pueden existir otros mecanismos de convergencia que pueden ser importantes. Por lo que en la práctica sería mejor interpretar el valor de b como un resumen del efecto neto de toda una serie de fuerzas con efectos distintos y posiblemente contrapuestos sobre la evolución de la desigualdad. Como lo proponen Barro y Sala-i-Martín (1992), sería interesante descomponer la convergencia observada en varios factores que reflejen distintos mecanismos de convergencia. Este ejercicio fue realizado por De la Fuente (1995), quien intentó cuantificar la contribución a la convergencia de la difusión tecnológica y los rendimientos decrecientes entre los países de la OCDE, los resultados indicaron que ambos mecanismos parecen ser operativos, pero que el primero es mucho más importante cuantitativamente que el segundo. En el caso de los países centroamericanos, sería también necesario un estudio más a fondo sobre el rol que juegan distintos mecanismos tales como, los flujos comerciales, la difusión tecnológica y posibles externalidades, antes de hacer conclusiones sobre los determinantes del crecimiento económico y la convergencia.

\subsection{Convergencia estocástica}

Una manera de probar la convergencia regional consiste en analizar las propiedades de las series de tiempo de los productos per capita relativos. Si bien éstos no siguen un proceso estacionario con media cero debido a que los países analizados seguramente se encuentran lejos de sus respectivos estados estacionarios y además estos últimos pueden ser diferentes, se sigue la propuesta de Carlino y Mills de permitir la existencia de procesos de convergencia condicional. De esta manera, en los tests de 
raíz unitaria utilizados se permite la existencia de ordenada al origen y de tendencia.

Siguiendo este enfoque, se presentan en el cuadro 4 los resultados de correr la siguiente versión ampliada de la ecuación 5 que incluye la variable de tendencia para cada uno de los países analizados:

$$
D\left(Y_{i 1}-Y_{m l}\right)=a_{i}+b_{i} t+h_{i}\left(Y_{i t-1}-Y_{m t-1}\right)+S z_{j} D\left(Y_{t-j}-Y_{m l-j}\right)+e_{i t}
$$

en donde $\left(\mathrm{Y}_{\mathrm{it}}-\mathrm{Y}_{\mathrm{ml}}\right)$ es la diferencia entre el logaritmo producto per cápita de cada país y el logaritmo de la región para el país $i$ en el año $t$ y el número de rezagos de la variable diferenciada $(k)$ se selecciona partiendo de 8 reduciendo este valor en la medida en que el estadístico t del último rezago sea menor (en valor absoluto) que 1.6 (siguiendo a Zivot and Andrews (1992)) con el propósito de que los residuos $e_{i t}$ no presenten autocorrelación.

Como se mencionó anteriormente, la presencia de raíz unitaria indica que la evolución de las series temporales es afectada por shocks permanentes. En el caso contrario de no raíz unitaria, los shocks son transitorios, por lo que se podrá hablar de existencia de convergencia. Para detectar si las series de producto per cápita son estacionarias, se utiliza la prueba aumentada de Dickey-Fuller.

En este caso, la hipótesis nula es que $\left(\mathrm{Y}_{\mathrm{ii}}-\mathrm{Y}_{\mathrm{m}}\right)$ no es estacionaria o posee raíz unitaria $\left(\mathrm{H}_{\mathrm{n}} \mathrm{h}_{\mathrm{i}}=0\right)$. La verificación de la hipótesis se lleva a cabo probando la significancia del coeficiente $h_{\mathrm{i}}$. El estadístico $t$ de dicho coeficiente no se compara con la $t$ de Student tabular, sino con los valores críticos proporcionados por unas tablas especiales construidas por Dickey-Fuller y algunos autores, a través de simulaciones de Monte Carlo.

\section{TABLA 4}

Resultados del test de ADF

\begin{tabular}{|l|l|r|r|r|r|}
\hline País & $\mathrm{K}$ & Constante & Tend & $\mathrm{Y}_{1-1}$ & \multicolumn{1}{c|}{ ADF } \\
\hline Costa Rica & 1 & 0.126 & 0.0052 & 0.273 & $-5.060^{* * *}$ \\
El Salvador & 8 & -0.013 & 0.0003 & -0.345 & 0.216 \\
Guatemala & 6 & -0.006 & 0.0006 & -0.285 & -2.182 \\
Honduras & 0 & -0.050 & 0.0003 & -0.099 & -1.850 \\
Nicaragua & 8 & 0.131 & -0.0064 & -0.308 & -0.692 \\
\hline *** significativo al 1\% $\%$ \\
Número total de observaciones: 46 para cada país \\
\hline
\end{tabular}


Como puede observarse en el mencionado cuadro, la hipótesis nula de existencia de raíz unitaria no puede ser rechazada en ningún país, excepto Costa Rica, al nivel de 1 por ciento. Por lo tanto, no se puede aceptar la existencia de convergencia estocástica. A pesar de que lo anterior constituye evidencia contraria a la existencia de un proceso de convergencia debido a la persistencia de los shocks sobre los productos per cápita, es importante tener en cuenta que el test utilizado se caracteriza por su bajo poder de rechazo de hipótesis nulas falsas, problema que se agrava a medida que disminuye el tamaño de la muestra.

Los problemas mencionados arriba han conducido a probar la hipótesis de convergencia a partir de otro método. Este último utiliza las series temporales para examinar las distancias que existen entre los niveles del producto real per cápita de cada uno de los países y el nivel del país "leader", es decir, el país que presenta el más alto nivel del PIB real per cápita. Si las distancias tienden a disminuir en el tiempo, se puede concluir que efectivamente existe convergencia aunque parezca más apropiado hablar de acercamiento en el marco de este método.

La ecuación a estimar por medio de Mínimos Cuadrados No Lineales (MCNL) es de la siguiente forma:

$$
Y_{i, 1}-Y_{j, t}=a^{*} e^{-d t}+e_{i, 1}
$$

Donde $Y_{i, t}-Y_{j, t}$, la diferencia entre los niveles logarítmicas del PIB real per cápita de los países $\mathrm{j}$ e i describe una función de tipo exponencial decreciente. El término a representa la constante, mientras que el coeficiente $d$ indica la pendiente. Entre más elevado sea d, más rápido se reducen la distancia entre el PIB per cápita de los países j e i. La amplitud del coeficiente de $\mathrm{d}$ es totalmente independiente de la del coeficiente a. Se considera que existe acercamiento entre los países i e j si el coeficiente $\mathrm{d}$ es positivo y significativo desde el punto de vista estadístico.

\section{TABLA 5}

\section{Estimación de la ecuación (8) para el período 1952- 1995}

\begin{tabular}{|c|c|c|c|c|}
\hline Variable & $Y_{o r}-Y_{z u}$ & $Y_{o r}-Y_{m b}$ & $Y_{o x}-Y_{b=}$ & $Y_{a r}-Y_{d x}$ \\
\hline $\begin{array}{l}\text { a. } \\
\text { Slal-T }\end{array}$ & $\begin{array}{l}0.091 \\
(4.1)\end{array}$ & $\begin{array}{l}0.710 \\
(2.9)\end{array}$ & $\begin{array}{l}0.168 \\
(3.3)\end{array}$ & $\begin{array}{l}0.0311 \\
(2.4)\end{array}$ \\
\hline$\delta_{\text {Stal. } T}$ & $\begin{array}{l}-0.017 \\
(-5.8)\end{array}$ & $\begin{array}{r}-0.016 \\
(-5.3)\end{array}$ & $\begin{array}{l}-0.006 \\
(-2.9)\end{array}$ & $\begin{array}{r}-0.06 \\
(-7.1)\end{array}$ \\
\hline 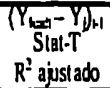 & $\begin{array}{l}0.545 \\
(5.5) \\
0.880\end{array}$ & $\begin{array}{l}0.666 \\
(5.1) \\
0.915\end{array}$ & $\begin{array}{l}0.770 \\
(10.1) \\
0.905\end{array}$ & $\begin{array}{l}0.686 \\
(5.8) \\
0.944\end{array}$ \\
\hline Dev-estandar & 0.032 & 0.1135 & 0.01035 & 0 \\
\hline Dur-wel. & T.69 & T.7T & 7.70 & TST \\
\hline
\end{tabular}

Número tolal de observaciones: 4 para cada serie 
La estimación de la ecuación (8) con los datos del PIB per cápita para los países centroamericanos durante el período 1952 - 1995 es presentada en la tabla (5). El análisis se efectúa a partir del logaritmo del PIB per cápita de Costa Rica, que es el país más rico durante el período. Luego, se obtienen las distancias, restando del PIB per cápita del Leader el valor que corresponde a cada uno de los países en cada año. El coeficiente d es negativo y significativo, lo que indica que los países no se acercaron a Costa Rica y que las distancias de los países con respecto a Costa Rica han ido creciendo en el tiempo. Eso es compatible con el resultado de la regresión a la Barro que indica la no convergencia absoluta del PIB per cápita de los países centroamericanos. Además, se estimó el modelo para los mismos subperíodos mencionados en el análisis de sigma convergencia; los coeficientes siguen siendo negativos y significativos. Ha de notarse que las distancias observadas durante el período precedente $\left(\left(Y_{\text {leadi }}-Y_{j}\right)_{t-1}\right)$ constituyen un elemento explicativo importante de las distancias observadas durante el período corriente de la mayoría de los países.

\section{Conclusiones}

Las grandes disparidades observadas empíricamente en la renta de los distintos países así como el deseo de explicar dichas diferencias y su evolución han generado un profundo interés por la teoría de crecimiento y han hecho de ésta un área profundamente tratada por la investigación económica. Al mismo tiempo, dentro de la literatura de crecimiento, el debate de la convergencia ha captado gran parte de la atención.

Una preocupación recurrente en los estudios de convergencia ha sido en qué medida los datos respaldan la existencia de convergencia absoluta, es decir, si todos los países convergen a un mismo estado estacionario, siendo los más pobres los que se acercan más rápidamente al mismo. La falta de evidencia a favor de esta hipótesis dio lugar a la consideración del concepto de convergencia condicional, que implica que las economías tienden a crecer más de prisa cuanto más alejadas se encuentran de su propio estado estacionario.

En la investigación de la convergencia condicional es obligada la referencia a los artículos de Barro y Sala-i-Martin. Las estimaciones llevadas a cabo por estos autores no permiten rechazar la hipótesis de convergencia aunque implican que el proceso de convergencia es sorprendentemente lento. Estos resultados se mantienen en múltiples trabajos posteriores cuando se consideran diferentes muestras de países o regiones. En los últimos años, han aparecido algunos artículos en los que se trabaja con la hipótesis de que la convergencia estimada a la fecha es lenta por una mala especificación del modelo. Estos autores encuentran evidencia 
a favor de una convergencia más rápida, pero de cada país hacia su estado estacionario.

Las limitaciones tanto a nivel teórico como a nivel de la metodología de estimación del enfoque tradicional de convergencia, han dado lugar al surgimiento de nuevos enfoques para comprobar la hipótesis de este fenómeno entre países o regiones. Dentro de éstos se incluyen las estimaciones de panel, el análisis de series de tiempo y la propuesta de Quah sobre el análisis de la distribución del producto per cápita.

En el caso de los países centroamericanos, los resultados indican la presencia de grandes diferencias entre ellos, diferencias que no tienden a reducirse con el paso de los tiempos, como lo demuestran el análisis de algunos indicadores como la desviación estándar de los productos per cápita y el análisis de la tendencia de las distancias de los países con respecto a Costa Rica.

Efectivamente, la desviación estándar de los productos y las distancias entre países se incrementaron entre los años 1950 y 1995. Estos indicadores han mantenido su tendencia creciente durante los años sesenta, setenta y noventa. Esto indica que la integración económica no ha contribuido a reducir las disparidades entre países, a pesar de la participación creciente del comercio intrarregional dentro de las exportaciones totales durante estos períodos. Por ende, el proceso de crecimiento que experimentaron estos países a lo largo de las cuatro décadas analizadas implica una distribución de productos per cápita que no tiende a concentrarse sino que permite hablar de tres grupos en términos de PIB per cápita: Costa Rica se halla en el nivel más alto; en una posición intermedia están El Salvador y Guatemala; mientras que Honduras y Nicaragua tienen el PIB per cápita menor de la región.

No existe evidencia de convergencia estocástica en la región. Sólo para Costa Rica se pudo rechazar la hipótesis de raíz unitaria. Eso indica que los shocks que afectan a los ingresos per cápita poseen impactos permanentes, es decir, el PIB per cápita de un año dado es el reflejo de una secuencia de shocks experimentados por los países durante su trayectoria económica. Esto es particularmente relevante para El Salvador y Nicaragua, debido a los shocks socio-políticos experimentados durante los ochenta.

Los resultados obtenidos no apoyan la hipótesis de $\beta$ convergencia absoluta en Centroamérica. Para el período de estudio, las mayores tasas de crecimiento corresponden a los países de PIB per cápita iniciales más altos. Se rechaza la existencia de la convergencia hacia un mismo estado estacionario. Lo que implica que las características estructurales juegan un papel importante en la determinación de las disparidades entre los 
niveles de ingreso de estos países.

Al tener en cuenta la existencia de distintos estados estacionarios, mediante la inclusión de efectos fijos, no se puede rechazar la hipótesis de convergencia entre los países centroamericanos. Los resultados reflejaron una velocidad de convergencia mayor al tradicional $2 \%$. Lo que hace sospechar que pueden existir otros mecanismos diferentes a los rendimientos decrecientes que aceleran el proceso de convergencia.

Sin embargo, aunque se ha encontrado una elevada estimación, esto no implica que las diferencias entre países de la región tienden a eliminarse. Estos resultados permiten derivar algún tipo de implicación de política económica regional. En la medida que los estados estacionarios de los países difieren, se hacen necesarias medidas de tipo estructural que traten de eliminar las distancias entre los mismos. A nivel centroamericano, se han registrado avances significativos en asuntos como las normas del intercambio intrarregional. Sin embargo, se tiene que trabajar en otros asuntos tales como la armonización de políticas macroeconómicas, laborales, sociales e institucionales de tal modo que la integración regional pueda constituirse en una herramienta eficaz de apoyo y complemento a la acción de cada país.

Los resultados de la convergencia condicional parecen favorecer la utilización de modelos de crecimiento con rendimientos marginales decrecientes y constantes a escala para estudiar el crecimiento de los países centroamericanos. Sin embargo, los problemas asociados a la robustez estadística y a la estimación del coeficiente a partir de un modelo basado en el comportamiento de una economía representativa, no permiten discriminar entre los modelos de crecimiento neoclásicos y los de crecimiento endógeno.

\section{Referencias bibliográficas}

Barro, R. (1991). "Economic Growth in a Cross Scction of Countrics." Quarterly Journal of Economics CVI (2), pp. 407-43.

Barro, R. (1997), "Determinants of Economic Growth: A cross-country cmpirical study", Harvard University, Development Discussion Paper № 579, April.

Barro, R. and X. Sala i Martin (1990). "Economic Growth and convergence across the United States." NBER Working Paper, num. 3419, August.

Barro, R. and X. Sala i Martin (1991). "Convergence across States and Regions." Brookings Papers on Economic Activity 1, 1991, pp. 107-82.

Barro, Robert J. and Xavicr Sala-i-Martin (1992a). "Convergence", Journal of Political Economy, Vol.100, No.2, April.

Barro, R. and X. Sala i Martin (1992h). "Regional Growth and Migration: A JapanUnited States Comparison." Journal of the Japanese and International Economies 6, pp. 312-46. 
Barro, Robert J. and Xavier Sala-i-Martin (1995). Economic Growth, The MIT Press, Cambridgc, Massachuselts.

Baumol, W. (1986). "Produclivity Growth, Convergence and Welfare: What the LongRun Data Show."American Economic Review 76(5), pp. 1072-85.

Bernard, Andrew B. and Steven N. Durlauf 1996). "Interpreting tests of the convergence hypothesis", Journal of Econometrics, 71.

Cáceres, Luis René y Nuñez-Sandoval, Óscar (1998). "Convergence in Central America", Inter-American Development Bank, Intcrnational Trade and Finance Association, Spring 1998 Meetings.

Canova, F. and A. Marcet (1995). "The Poor Stay Poor: Non-convergence across Countries and Regions." CEPR Discussion Paper, no. 1265.

Carlino, Gerald A. and Leonard Mills (1996). "Tesling neoclassical convergence in regional incomes and earnings", Regional Science and Urban Economics, 26.

Coulombe, S. and F. Lee (1993). "Regional Economic Disparitics in Canada." Mimco, University of Ottawa.

Coulombe, Serge (1997) "Les disparités regionales au Canada: diagnostic, tendances et leçons pour la politique ćconomique" Document de travail 18 ,Département de science économique, Université d'Ottawa, Novembre.

Coulombe, Serge and Lee, Frank (1996) "Long Run Perspective on Canadian regional convergence", working paper 11, Department of Economics, University of Ottawa, November.

De la Fuente, A. (1994a). "Crecimiento y convergencia: Un panorama selectivo de la cvidencia cmpirica", Cuadernos Económicos de Información Comercial Española, 58, pp.23-70.

De la Fuente, A. (1994b). "Crecimiento y convergencia", Crecimiento y convergencia regional en España y Europa, Instituto de Análisis Económico, 2, pp.199-247.

De la Fuente, A. (1995). "Catch-up, Growth and Convergence in the OECD." CEPR Discussion Paper no. 1274.

De la Fuente, A. (1996a). "Convergencia y otras historias: economía regional desde una perspectiva neoclásica."Revista de Economía Aplicada IV, 10, Primavera, pp. 5-64.

De la Fuente, A. (1996b). "On the sources of convergence: A close look at the Spanish regions." CEPR Discussion Paper No. 1543.

De la Fuente, A. (1996c). "Notas sobre la cconomía del crecimiento II: ¿Convergencia?". Instituto de Análisis Económico, Universidad Autónoma de Barcelona.

De la Fucnte, A. (1998). "What kind of regional convergence?" CEPR Discussion Paper no. 1924.

De la Fuente, A. (2000). "Convergence across countries and regions: theory and empirics, Instituto de Análisis Económico (CSIC), Working paper 447.00, January.

De Long, J.B. (1988), "Productivity Growth, convergence and welfare: Comment" American Economic Review, vol.78, pp.1138-1154.

Dolado, J., J. M. González-Páramo and J. M. Roldán (1994). "Convergencia Económica entre las Provincias Españolas: Evidencia Empirica (1955-1989)." Moneda y Crédito 198, pp. 81-131.

Dowrick, S. y D. T. Nguyen (1989). "OECD Comparative Economic Growth 1950-85: Catch-up and Convergence." American Economic Review 79(5), Dec. 1989, pp. $1010-$ 1030.

Durlauf, Steven N. and Dann T. Quah 1998): "The new empirics of economic growth", University of Wisconsin, Madison and LSE, January.

Evans, P. and G. Karras (1996). "Do economies converge? Evidence from a panel of US statcs." Review of Economics and Statistics, 384-88.

Gorostiaga, A. (1998). “CCómo afectan el capital público y el capital humano al creci- 
miento? Un análisis para las regiones españolas en el marco neoclásico." Mimeo, CEMFI, Madrid (forthcoming in Investigaciones Económicas).

Guin-Siu, Maria Teresa (1999), "Growth in Latin America", mimeo.

Islam, N. (1995) "Growth Empirics: A panel data approach" Quaterly Journal of Economics.

Lamo, Ana (2000), "On convergence empirics: Some evidence for Spanish regions", European Central Bank.

Lefcbvre, Mario. (1994) "Les provinces canadicnnes et la convergence: unc ćvaluation empirique" Document de travail 94-10. Banque du Canada, Novembre.

Lucas, R. (1988). "On the Mechanics of Economic Development." Journal of Monetary Economics 22(1), pp. 3-42.

Mankiw, G., D. Romer and D. Weil (1992). "A Contribution to the Empirics of Economic Growth." Quartely Journal of Economics CVII(2), pp. 407-37.

Marcet, A. (1994). "Los pobres siguen siendo pobres: Convergencia entre regiones y países, un análisis bayesiano de datos de panel," en Crecimiento y convergencia regional en España y Europa, Vol. II. Instituto de Análisis Económico, Barcelona.

Neven, D and C. Gouyette (1995). "Regional convergence in the European Community." Journal of Common Market Studies, 33, pp. 47-65.

Quah, Danny T. (1993): "Galton's fallacy and tests of the convergence hypothesis", The Scandinavian Journal of Economics, Vol. 95, No.4.

Quah, Dann T. (1995a): "Convergence empirics across economies with some capital mobility“, Discussion Paper No.257, Centre for Economic Performance, August.

Quah, Dann T. (1995b): "Regional convergence clusters across Europe", Discussion Paper No.274, Centre for Economic Performance, December.

Quah, Dann T. (1996a): "Empirics of economic growth and convergence", European Economic Review, Vol.40, No.6, June.

Quah, Dann T. (1996b): "Twin peaks: growth and convergence in models of distribution dynamics", The Economic Journal, Vol.106, No.437, July.

Quah, Dann T. (1997): "Empirics for growth and distribution: stratification, polarization and convergence clubs", Discussion Paper No.324, Centre for Economic Pcrformance, January .

Rebclo, Sergio (1991), "Long-run policy analysis and long-run growth", Journal of Political Economy, 99,3 (Junc), pp.500-521.

Rivera-Batiz, L.A. y Romer, P. (1991) "Economic Integration and Endogenous Growth", Quarterly Journal of Economics, 106(2), pp.531-555.

Romer, P. (1990). "Endogenous Techniçal Changc." Journal of Political Economy 98, pp. S71-S102

Sala-i-Martin, Xavier (1990), On growth and States. Ph.D. dissertation (Harvard University Cambridge, MA).

Sala-i-Martin, Xavier (1994), Apuntes de crecimiento económico, Editorial Antoni Bosch, Barcelona, Primera edición.

Sala i Martin, X. (1995). "Rcgional cohesion: evidence and theorics of regional growth and convergence." European Economic Review 40, pp. 1325-52.

Sala i Martin, X. (1996). "The classical approach to convergence analysis". The Economic Journal, vol. 106, Num. 437, July.

Shioji, E. (1996). "Regional Growth in Japan." CEPR Discussion Paper no. 1425.

Shioji, E. (1997a). "Convergence in pancl data: cvidence from the skipping estimation." Mimco, Universitat Pompeu Fabra, Barcclona.

Shioji, E. (1997b). "It's still 2\%. Evidence of convergence from 116 years of the US statcs pancl data." Mimco, Univcrsitat Pompeu Fabra, Barcclona. 
Solow, R. (1956). “A Contribution to the Theory of Economic Growth." Quarterly Journal of Economics LXX(1), pp. 65-94.

Solow, R. (1957). "Technical Change and the Aggregate Production Function," Review of Economics and Statistics, 39, pp. 312:20.

Utrera, Gastón Ezequiel and Javier Adolfo Koroch (1999): "Regional convergence in Argentina: empirical evidence", The XIIth World Congress, International Economic Association, August.

Utrera, Gastón Ezequiel (1999), “El crecimiento económico en Latinoamérica”, Instituto de Investigaciones Económicas- Bolsa de Comercio de Córdoba.

Zivot, Eric and Donald W. K. Andrews 1992): "Further evidence on the great crash, the oil-price shock, and the unit-root hypothesis", Journal of Business \& Economic Statistics, Vol.10, No.3, July.

\section{Notas}

1. Sala-i-Martin (1990), Barro y Sala-i-Martin (1991, 1992a) y Mankiw, Romer y Weil (1992) desarrollaron el conceplo de convergencia condicional para contraponerlo al concepto de convergencia b absoluta utilizado hasta entonces, ncgando que la evidencia empírica pudiera ser utilizada en contra del modelo neoclásico.

2. De la Fuente, Ángel (1996c). "Notas sobre la economía del crecimiento II: ¿Convergencia?". Instituto de Análisis Económico, Universidad Autónoma de Barcelona, pag.60.

3. Quah, Danny T (1995), “Empirics for Economic Growth and Convergence", LSE Economics Department and CEP, pág 15.

4. De la Fuente, Angel, Op. Cit. Pág 60. Para un mayor desarrollo de este tema, ver Sala-i-Martin (1994).

5. Utrera, Ezequiel Gastón (1999), "El crecimiento económico en Latinoamérica". Instituto de Investigaciones Económicas, Universidad Nacional de Córdoba, pág 6.

6. Tampoco Cáceres (1998) pudo rechazar la hipótesis de convergencia estocástica en estos paises para el período 1920-1984, usando datos del PIB per cápita de BulmerThomas (1986).

7. La ecuación corresponde a la versión estimada por Mario Lefebvre (1994) para las provincias de Canadá. 